\title{
Simple and Eco-friendly Synthesis of Glycosides bearing triazolo[3,4-b][1,3,4]thiadiazole moiety linked through $\mathrm{N}$ atoms
}

\author{
Sunil Kumar Bhat* Jagjeet Singh \\ Department of Chemistry G.G.M. Science College Jammu.
}

\begin{abstract}
Abstrasct: There is a vast variety of naturally occurring glycosides which have marked pharmacological properties. These glycosides have widely diversed functional groups modifications which result in influencing pharmacological performance of corresponding glycosides. The 3,6-disubstituted-s-triazolo[3,4b][1,3,4]thiadiazoles were glucosidated with 2,3,4,6tetra-o-acetyl $\alpha$ D glulopryanosyl bromide using simple methodologies. The compounds obtained in good yield in a 80-90 minutes.
\end{abstract}

Keywords: anti helmintic, anti hypertensive, thiosemicarbozes, triozoles, thiadiazoles

\section{Introduction}

The N-heterocycles are known for their potent biological activities. Among them the 1,2,4-triazoles and N-bridged heterocyclic have received a considerable attention due to their strong pharmacological properties[15]. A triazolo nucleus appear to be very important for its biological potency. Likewise in various heterocyclic products a 1,3,4-thiadiazole ring also possesses a diverse biological activities due to $-\mathrm{N}-\mathrm{C}=\mathrm{S}$ linkage, the importance of which has been well stressed in many pesticides. Further 2-amino-1,3,4-thiazoles may be viewed as cyclic analogue of thiosemicarbazone, the physiological properties of which have been well established. The triazolo[3,4-b][1,3,4]-thiadiazoles, thiosemicarbazide[6] and biguanides[7] which posses diverse biological activities such as antimicrobial, bacteriocidal, anti inflammatory antiviral, anti hypertensive anti helmintic and analgesic [8-25].

The glycosides are found in a wide variety of natural products. The carbohydrate moiety in these glycosides is generally a mono or disaccharide and less frequently a higher oligosaccharide. These glycosides are attached to aglycan part through C-1 of carbohydrate and N-part of amine. Numerous glycosides are used as drugs and have a wider therapeutic applications [26-29]. Most of these glycosides are optically active.

\section{Results and Discussion}

The series of glycosides bearing triazolo[3,4-b][1,3,4]thiadiazole moiety linked through $\mathrm{N}$ atoms was synthesized. The $\mathrm{N}$-glycosides were condensed with mercapto amino triazoles in a microwave oven in presence of DMF and $\mathrm{TsOH}$.

General scheme involves glucosidation of 3,6-disubstituted-s-triazolo[3,4-b][1,3,4]-thiadiazoles with 2,3,4,6-tetra-O-acetyl $\alpha$-D glucopyranosyl bromide having N-glucoside linkage. A mixture of hydrazine hydrate, water and $\mathrm{CS}_{2}$, on refluxing yielded thiocarbohydrazide (1). This on heating with carboxylic acid gave 4aminomercapto-3-alkyl-s-triazole(2). The (2) on treatment with aromatic aldehyde in dry benzene using DeanStark apparatus and catalytic amount of TsOH yielded 3,6-disubstituted-s-triazolo[3,4-b][1,3,4]-thiodiazoles (Scheme - I).

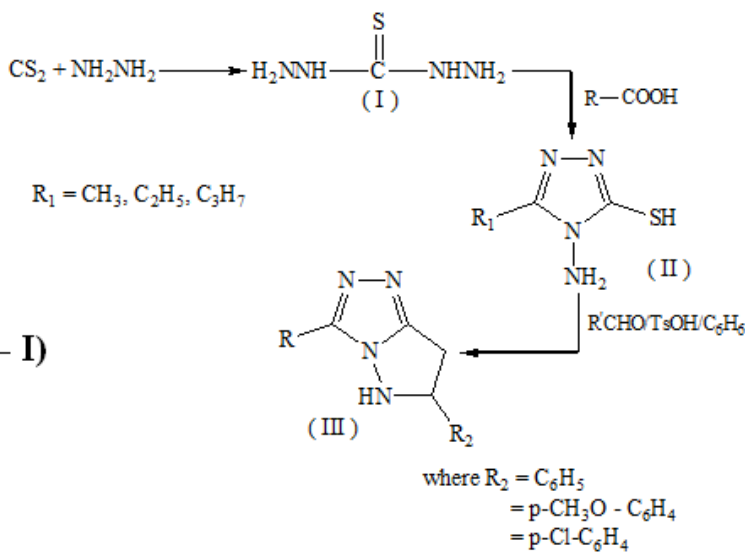


3,6-Disubstituted-s-triazole [3,4-6][1,3,4]-thiadiazoles (2) on treatment with 2,3,4,6,tetra-o-acetyl- $\alpha$-Dglucopyranosyl bromide (3) gave substituted $\mathrm{N}$-glucosidated-s-triazolo[3,4-6][1,3,4]-thiadiazoles (4) [SchemeII].<smiles>[R]c1nnc2n1NC([R])[S-]2</smiles>

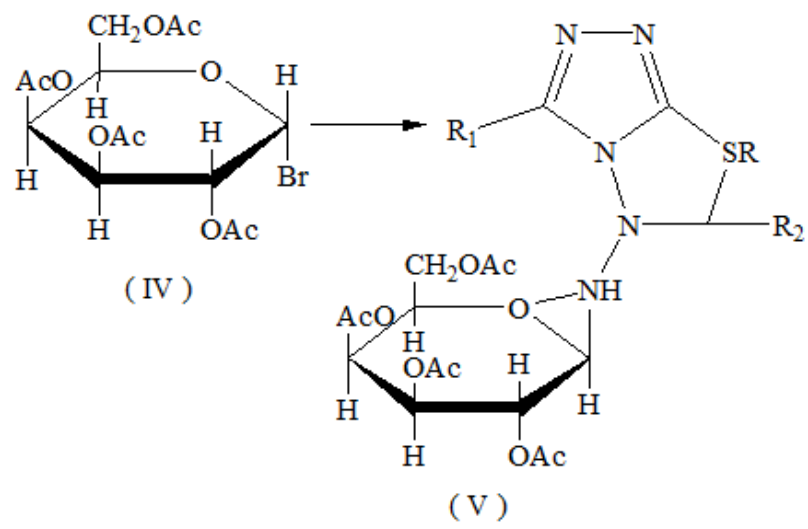

(Scheme-II)

The compounds show N-H stretching band between $3150-3100 \mathrm{~cm}^{-1}$. The $\mathrm{C}=\mathrm{O}$ stretching band of acetyl carbonyl occurs at $1770-1750 \mathrm{~cm}^{-1}$. In the NMR of the compounds show the C-H as singlet at $\delta$ value of 5.65 to 5.80 .

\begin{tabular}{|l|l|l|l|}
\hline Group & \multirow{2}{*}{ Time in hr. } & \multirow{2}{*}{ Yield (\%) } \\
\hline $\mathrm{R}_{1}$ & $\mathrm{R}_{2}$ & & \\
\hline $\mathrm{CH}_{3}$ & $\mathrm{C}_{6} \mathrm{H}_{5}$ & $1 \mathrm{hr}-20 \mathrm{~min}$. & 60 \\
\hline $\mathrm{C}_{2} \mathrm{H}_{5}$ & p- $\mathrm{CH}_{3} \mathrm{O}-\mathrm{C}_{6} \mathrm{H}_{4}$ & $1 \mathrm{hr}-25$ min. & 62 \\
\hline $\mathrm{C}_{3} \mathrm{H}_{7}$ & p- $\mathrm{CH}_{3} \mathrm{O}-\mathrm{C}_{6} \mathrm{H}_{4}$ & $1 \mathrm{hr}-22$ min. & 65 \\
\hline $\mathrm{CH}_{3}$ & p-Cl- $\mathrm{C}_{6} \mathrm{H}_{4}$ & $1 \mathrm{hr}-20 \mathrm{~min}$. & 63 \\
\hline $\mathrm{CH}_{3}$ & p-Br- $\mathrm{C}_{6} \mathrm{H}_{4}$ & $1 \mathrm{hr}-27 \mathrm{~min}$. & 67 \\
\hline $\mathrm{C}_{2} \mathrm{H}_{5}$ & p-Cl- $\mathrm{C}_{6} \mathrm{H}_{4}$ & $1 \mathrm{hr}-28 \mathrm{~min}$. & 66 \\
\hline $\mathrm{CH}_{3}$ & p-2,4-dichlorophenyl & $1 \mathrm{hr}-35 \mathrm{~min}$. & 68 \\
\hline $\mathrm{CH}_{3}$ & p- $\mathrm{CH}_{3} \mathrm{O}-\mathrm{C}_{6} \mathrm{H}_{4}$ & $1 \mathrm{hr}-30 \mathrm{~min}$. & 69 \\
\hline
\end{tabular}

\section{Experimental}

Preparation of thiocarbazide (1). A mixture of $156.25 \mathrm{ml}(80 \%)$ of hydrazine hydrate and $95 \mathrm{ml}$ of water was stirred well for 10 minutes and $38 \mathrm{ml}$ of $\mathrm{CS}_{2}$ as added in small increments with constant stirring. After this the reaction mixture was allowed to reflux for 2-3 hours. The flask was cooled and the reaction mixture was filtered and recrystallised with hot water, when shinning while crystals with m.pt $169{ }^{\circ} \mathrm{C}$ were obtained.

\section{Preparation of 4-Amino-5-mercapto-3-alkyl-s-triazole (II)}

Thiocarbazide and carboxylic acid (1:10 molar ratio) was refluxed for three to four hours. When the crystals started separating out. These were recrystallised with hot water.

\section{Preparation of 3-Alkyl-6-phenyl-5,6-dihydro-s-triazolo[3,4-6][1,3,4] thiadiazoles (III)}

The equimolar mixture of compound II benzaldehyde and TsOH were taken in a flask and to it $100 \mathrm{ml}$ of dry benzene was added. The contents were refluxed for 10 hours using Dean-Stark apparatus, to remove the traces of water formed during the course of reaction. The reaction was monitored with thin layer chromatography of the eloquates taken out of reaction mixture from time to time. After the completion of reaction, the reaction mixture was cooled and treated with water. Organic layer was separated dried and the solvent was evaporated. The residue was recrystallised using ethanol.

Preparation of 3-Alkyl-6(H)-6-phenyl-5-[N-2,3,4,6-tetra-o-acetyl-3-D-glucopyranosyl)-s-triazole[3,4-6] $[1,3,4]$-thiadiazole $(\mathrm{V})$

A mixture of compound(III) ( $0.05 \mathrm{~mol})$ sodium hydride (50\% suspension in mineral oil) $(0.05 \mathrm{~mol})$ were stirred in $225 \mathrm{ml}$ of acetonitrile at room temperature for one hour. Tetra-o-acetyl- $\alpha$-D-glucopyranosyl bromide(IV) [Prepared through the methods reported in literature] was added to it in 1:1 mole ratio in small quantities and allowed to stir for another 25-30 minutes to complete the reaction. The mixture was filtered through celite and washed with $\mathrm{CHCl}_{3}$. The product were recrystallised on silica gel (10-230 mesh) on column chromatography using graded solvents.

All the solvent were of LR and were obtained from Merck. The elemental analysis of the compounds was performed on CHN elementary (Analysen System Gm6H, Germany) The elemental analysis (C,H,N) of compounds was found within a limit of $\pm 0.5 \%$ theoretical values of $0.5 \mathrm{~mm}$ thickness. The TLC plates were prepared from silica gel G Merck Iodine chamber was used for visualization of TLC spots. The FT-IR spectrum 
Simple and Eco-friendly Synthesis of Glycosides bearing triazolo [3,4-b][1,3,4]thiadiazole moiety

was recorded in $\mathrm{KBr}$ pellets on a Perkin Elmer FT-IR spectrometer. The melting points were determined on Yorko melting point apparatus and thermometer was uncorrected. ${ }^{1} \mathrm{H}$ NMR spectra was recorded in $\mathrm{CDCl}_{3}$ with TMS as internal standard on Bruker NMR spectrophotometer operating at $200 \mathrm{MHz}$ chemical shifts are expressed in $\delta$-values (in ppm) relative to TMS as internal standard.

(Va). 3-Methyl-6(H)-6-phenyl-5-[N-2,3,4,6-tetra-o-acetyl- $\beta$-D-glucopyranosyl]-s-triazole[3,4,6][1,3,4]thiadiazole.

Colourless crystals, m.pt $178{ }^{\circ} \mathrm{C}$. IR(KBr) $\cup_{\max } 3100,1770,1585,1350,1245,690 \mathrm{~cm}^{-1} .{ }^{1} \mathrm{H} \mathrm{NMR}\left(\mathrm{CDCl}_{3}\right): 1.66$ $-1.80\left(\mathrm{~m}, 2 \mathrm{H}, \mathrm{COCH}_{3}\right), 2.10(\mathrm{~s}, 3 \mathrm{H}), 3.85-4.25\left(\mathrm{~m}, 5 \mathrm{H},-\mathrm{OCH}_{2}, 3^{\prime}, 4^{\prime}, 5^{\prime}-\mathrm{H}\right), 4.5-4.65\left(\mathrm{~m}, 1^{\prime}, 2^{\prime}-\mathrm{H}\right), 5.70(\mathrm{~s}, 1 \mathrm{H}$, 6-H), 7.70-7.80(m, 5H, Ar-H).

Anal. CHN(\%): Found C, 52.05; H, 5.07; N, 10.18. $\mathrm{C}_{24} \mathrm{H}_{28} \mathrm{~N}_{4} \mathrm{O}_{9}$ S.Calc.C, 52.55; H, 5.10; N, 10.21 .

(Vb). 3-Ethyl-6(H)-6-[(4'-methoxy)phenyl]-5[N-2,3,4,6-tetra-o-acetyl- $\beta$-D-glucopyranosyl]-striazolo[3,46][1,3,4]-thiadiazole.

Light grey coloured crystals, m.pt $162{ }^{\circ} \mathrm{C}$. IR( $\left.\mathrm{KBr}\right) \mathrm{u}_{\max } 3150,1790,1590,1360,1235,695 .{ }^{1} \mathrm{H}-\mathrm{NMR}\left(\mathrm{CDCl}_{3}\right): \delta$ $1.17(\mathrm{t}, 3 \mathrm{H}), 3.72(\mathrm{~s}, 3 \mathrm{H}), 4.15(\mathrm{q}, 2 \mathrm{H}), 3.96-4.55(\mathrm{~m}, 5 \mathrm{H}), 4.65-4.73(\mathrm{~m}, 2 \mathrm{H}), 7.54-8.10(\mathrm{~m}, 4 \mathrm{H})$.

Anal. CHN(\%) for $\mathrm{C}_{26} \mathrm{H}_{32} \mathrm{~N}_{4} \mathrm{O}_{10}$ S, Found: C, 52.60; H, 5.05; N, 9.38.Calcd.C, 52.72; H, 5.40; N, 9.45.

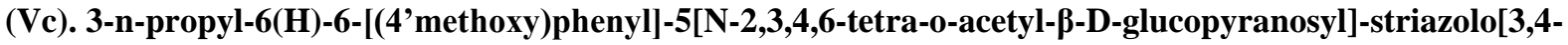
6][1,3,4]-thiadiazole.

Brown coloured crystals m.pt $=151{ }^{\circ} \mathrm{C} . \mathrm{IR}(\mathrm{KBr}): \mathrm{v}_{\max } 3130,1765,1560,1330,1230,670 .{ }^{1} \mathrm{H}-\mathrm{NMR}\left(\mathrm{CDCl}_{3}\right): \delta$ $1.20(\mathrm{t}, 3 \mathrm{H}), 1.90-2.00(\mathrm{~m}, 12 \mathrm{H}), 2.40-2.66(\mathrm{~m}, 4 \mathrm{H}), 3.7(\mathrm{~s}, 3 \mathrm{H}), 3.75-4.33(\mathrm{~m}, 5 \mathrm{H}), 4.55-4.67(\mathrm{~m}, 6 \mathrm{H}), 5.70(\mathrm{~s}, 1 \mathrm{H})$, 7.65-8.05(m, 4H).

Anal.Calcd. For $\mathrm{C}_{27} \mathrm{H}_{34} \mathrm{~N}_{4} \mathrm{O}_{10}$ S. Found: C, 53.40; H, 5.25; N, 9.02.Calcd. C, 53.46; H, 5.61; N, 9.24.

(Vd). 3-Methyl-6(H)-6-[4'Chlorophenyl]-5-[N-2,3,4,6-tetra-o-acetyl-3-D-glucopyranosyl]-s-triazolo[3,46][1,3,4]-thiadiazole.

Colourless crystals m.pt $163{ }^{\circ} \mathrm{C}$. IR( $\left.\mathrm{KBr}\right): \mathrm{v}_{\max } 3243,1760,1550,1320,1210,675 .{ }^{1} \mathrm{H}-\mathrm{NMR}\left(\mathrm{CDCl}_{3}\right): \delta 1.16(\mathrm{~s}$, $3 \mathrm{H}), 1.85-2.10(\mathrm{~m}, 12 \mathrm{H}), 2.30-2.50(\mathrm{~m}, 4 \mathrm{H}), 3.70-4.30(\mathrm{~m}, 5 \mathrm{H}), 5.60(\mathrm{~s}, 1 \mathrm{H}), 7.40-7.56(\mathrm{~m}, 4 \mathrm{H})$.

Anal.Calcd. For $\mathrm{C}_{24} \mathrm{H}_{27} \mathrm{O}_{9} \mathrm{~N}_{4} \mathrm{SCl}$. Found: C, 49.60; H, 4.52; N, 9.54.Calcd.C, 49.52; H, 4.64; N, 9.62.

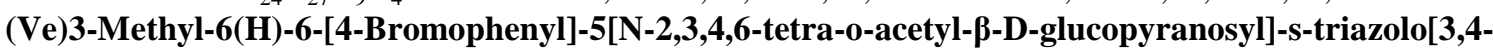

6][1,3,4]-thiadiazole.

Colourless crystals m.pt $161{ }^{\circ} \mathrm{C}$. IR( $\left.\mathrm{KBr}\right) \mathrm{v}_{\max } .3234,2993,1750,1565,1325,1220,680 \mathrm{~cm}^{-1} .{ }^{1} \mathrm{H}-\mathrm{NMR}\left(\mathrm{CDCl}_{3}\right)$ : $\delta 1.28(\mathrm{~s}, 3 \mathrm{H}), 1.80-2.00(\mathrm{~m}, 12 \mathrm{H}), 2.25-2.40(\mathrm{~m}, 4 \mathrm{H}), 3.70-4.20(\mathrm{~m}, 5 \mathrm{H}), 6.5-7.5(\mathrm{~s}, 1 \mathrm{H}), 7.20-7.36(\mathrm{~m}, 4 \mathrm{H})$.

Anal. Calcd. For $\mathrm{C}_{24} \mathrm{H}_{27} \mathrm{O}_{9} \mathrm{~N}_{4} \mathrm{SBr}$. Found: C, 46.10; H, 4.18; N, 8.76. Calcd. C, 45.96; H, 4.30; N, 8.92.

(Vf).3-Ethyl-6(H)-6-[4-Chlorophenyl]-5[N-2,3,4,6-tetra-o-acetyl-3-D-glucopyransoyl]-s-triazolo[3,4-

b] $[1,3,4]$-thiadiazole.

Colourless crystals m.pt $158{ }^{\circ} \mathrm{C}$. IR(KBr) $\mathrm{U}_{\max }: 3345,3040,1693,1080,670 \mathrm{~cm}^{-1} .{ }^{1} \mathrm{H} \mathrm{NMR}\left(\mathrm{CDCl}_{3}\right): \delta 1,16(\mathrm{t}$, $3 \mathrm{H}), 1.80-2.00(\mathrm{~m}, 12 \mathrm{H}), 3.98(\mathrm{q}, 2 \mathrm{H}), 4.15-4.40(\mathrm{~m}, 5 \mathrm{H}), 4.60-4.75(\mathrm{~m}, 2 \mathrm{H}), 7.50-7.80(\mathrm{~m}, 4 \mathrm{H})$.

Anal. Calcd. For $\mathrm{C}_{25} \mathrm{H}_{29} \mathrm{~N}_{4} \mathrm{O}_{9} \mathrm{SCl}$. Found: C, 50.38; H, 5.30; N, 9.26. Calcd. C, 50.32; H, 5.36; N, 0.38.

(Vg).3-Methyl-6(H)-6-[2',4'-dichlorophenyl]-5-[N-2,3,4,6-tetra-o-acetyl-p-D-glucopyranosyl]-striazolo[3,4-6][1,3,4]-thiadiazole.

Colourless crystals m.pt $182^{\circ} \mathrm{C}$.

$\operatorname{IR}(\mathrm{KBr}) \mathrm{U}_{\max }: 3291,1754,1595,1346,1050,680 \mathrm{~cm}^{-1} .{ }^{1} \mathrm{H} \mathrm{NMR}\left(\mathrm{CDCl}_{3}\right): \delta 1.74-2.12(\mathrm{~m}, 12 \mathrm{H}), 2.30(\mathrm{~s}, 3 \mathrm{H})$, $3.85-4.25(\mathrm{~m}, 5 \mathrm{H}), 4.54-4.71(\mathrm{~m}, 2 \mathrm{H}), 5.83(\mathrm{~s}, 1 \mathrm{H}), 7.35-7.93(\mathrm{~m}, 3 \mathrm{H})$.

Anal. Calcd. For $\mathrm{C}_{24} \mathrm{H}_{26} \mathrm{~N}_{4} \mathrm{O}_{9} \mathrm{SCl}_{2}$. Found: C, 46.82; H, 4.28; N, 9.17. Calcd.: C 46.70; H, 4.21; N, 9.07.

(Vh). 3-Methy-6(H)-6-[4'Methoxyphenyl]-5-[N-2,3,4,6-Tetra-o-acetyl- $\beta$-D-glucopyranosyl] -3-triazolo[3,4b] $[1,3,4]$-thiadiazole.

Colourless crystal m.pt $164{ }^{\circ} \mathrm{C}$. IR(KBr) $\mathrm{U}_{\max } 3291,1758,1575,1360,1220,685 \mathrm{~cm}^{-1} .{ }^{1} \mathrm{H} \mathrm{NMR}\left(\mathrm{CDCl}_{3}\right): \delta 1.70-$ $1.98(\mathrm{~m}, 12), 2.15(\mathrm{~s}, 3 \mathrm{H}), 2.35(\mathrm{~s}, 3 \mathrm{H}), 3.72-3.98(\mathrm{~m}, 5 \mathrm{H}), 4.6-4.7(\mathrm{~m}, 2 \mathrm{H}), 5.88(\mathrm{~S}, 1 \mathrm{H}), 7.80-7.95(\mathrm{~m}, 4 \mathrm{H})$.

Anal. Calcd. For $\mathrm{C}_{25} \mathrm{H}_{30} \mathrm{~N}_{4} \mathrm{O}_{10}$ S. Found: C, 5.18; H, 5.22; N, 9.62. Calcd: C, 51.92; H, 5.18; N, 9.68.

\section{References}

[1]. Z. Y. Zhang; X. Chen; Synthesis and antibacterial activity of 3-(4-pysidyl)-6-aryl-1,2,4-tetrazole[3,4-b]-[1,3,4]-thiadiazoles, Acta Chim Sinica, 49, 1991, 513-520.

[2]. N. Demcrbas, S. A. Karaoglu, A. Demirbas, K. Sanack, Synthesis and antimicrobial activities of some new 1-(5-phenyl amine-[1,3,4]thiadiazo-2-yl)methyl-5-oxo-[1,2,4]triazolo and 1-(4-phenyl-5-thioxo-[1,2,4]-triazolo-3-yl)methyl-5-oxo-[1,2,4] triazole derivatives. European J. of Med. Chem., 39, 2004, 793-804.

[3]. V. S. Palekar, A. J. Damle, S. R.Shukla, Synthesis and Antimicrobial activity of some novel bis-1,2,4-triazole[3,4-b]-[1,3,4]thiadiazole and bis-4-thiazolidinone derivatives from terephthalic dehydrazide. European J. of Med. Chem., 44, 2009, 5112-5116.

[4]. N. F. Swiss, A. A. Bahajaj and Elsherbin, J. Het. Chem., 23, 1986, 94.

[5]. N. Demirbas, A. Demirbas, S. A. Jaraoglu, E. Celik. Arkivoc, 2005(i), 75. K. C. Joshi and S. Giri, J. Indian Chem. Soc., 40, 1963, 42.

[6]. N. Tiwari B. Chaturvedi and Nizamuddin, Ind. J. Chem., 28B, 1989, 200.

[7]. J. Haglind, Inteen Congr Chemotherapy Proc, $3^{\text {rd }}$, Stuttgart, 1, 1963, 887; Chem. Abstr., 64, 1966, 16509e. 
[8]. M. M. Mansuri and C. M. John: Annual reports in Med. Chem., 26, 1991, 133

[9]. H. Johann, Z. Erich, B. Jan and De-C. Erike; J. Med. Chem., 35, 1992, 3016.

[10]. D. D. Guy, P. Daniel and Dorothy, Annual reports in Med. Chem., 24, 1989, 129.

[11]. M. Akira, N. Yuki, A. Atsuski, T. Motohiro and S. Takuma, J. Med. Chem., 34, 1991, 2917.

[12]. H. E. Renis, G. E. Underwood and J. H. Hunter: Antimicrob Agents Chemother. 675, 1967: J. J. Fox N. Miller and I. Wempen, J. Med. Chem., 9, 1966, 101.

[13]. L. Tai-Shun, H. P. William; J. Med. Chem., 21, 1978, 109.

[14]. L. Tai-Shun and Y. Jing-Hua, L. Mao-Chin, S. Zhi-Yi, C. Yung-Chi, H. William, I. B. George, G. Jerzy, G. Ismail, B. Vera, F. June-Sun and H. Guch-Djen; J. Med. Chem., 34, 1991, 693-701.

[15]. H. O. Helen and C. A. Richard; Annual reports in Med. Chem., 23, 1988, 332.

[16]. S. Yai and R. M. William; J. Med. Chem., 25, 1983, 544.

[17]. D. S. June and B. E. Daniel, Annual reports in Med. Chem., 27, 1992, 327. M. Noriaki T. Takayuki, S. Takuma, M. Akira and U. Tohru; J. Med. Chem., 34, 1991, 778-786.

[18]. R. R. Ganapathi, P.K. Gupta, D. A. Alexander, N. Kent Dalley Patricia A. Mc Kernan, P. D. Cook, G. C. Peter and K. R. Roland; J. Med. Chem., 27, 1984, 1389.

[19]. C. A. Richard; Annual reports in Med. Chem., 19, 1984, 313.

[20]. H. Hiroshi, W. Yohko, A. Toichi, M. Toshikkio, N. Yasukaru and M. Akira; J. Med. Chem., 35, 1992, 2881.

[21]. A. Toichi, M. Takanori, W. Yohko, Y. Toyofumi, M. Harukiko nd M. Akira; J. Med. Chem., 35, 1992, 2253.

[22]. C. B. David, P. C. Richard H. H. Theodore, R. P. Streling A. Gussie, M. S. William, C. M. Reinkle, B. K. Nomat and C. D. John; J. Med. Chem., 26, 1983, 1530.

[23]. R. R. Ganapathi, H. H. John, B. A. Lois, W. S. Robert K. R. Roland and L. T. Richard; J. Med. Chem., 18, 1975, 721

[24]. M. H. Elizabeth, D. K. Ganesh, B. L. Steven, K. R. Roland, A. A. Hassan and F. S. Donald; J. Med. Chem., 33, 1990, 2127

[25]. D. S. June and B. E. Daniel; Annual reports in Med. Chem., 27, 1992, 326.

[26]. J. H. Hoch, A Survey of Cardiac Glycosides and Genins, Charleston, University of South Carslina Press, 1961.

[27]. A. Hollman, Plants and Cardiac Glycosides Br. J. Heart, 54, 1985, 258-61.

[28]. K. A. Bose, M. S. Manhas, M. Gosh, M. Shah, V. S. Raju, S. S. Bari, S. N. Newaz, B. K. Banik, A. G. Choudhary and K. J. Barket, J. O. Chem. 56, 1991, 6971-6992.

[29]. M. M. Muzammi and C. M. John: Annual reports in Med. Chem., 22, 1987, 152. 